LIVER

\title{
Prognostic factors for survival in patients with early- intermediate hepatocellular carcinoma undergoing non- surgical therapy: comparison of Okuda, CLIP, and BCLC staging systems in a single Italian centre
}

\author{
A Grieco, M Pompili, G Caminiti, L Miele, M Covino, B Alfei, G L Rapaccini, G Gasbarrini
}

Gut 2005;54:41 1-418. doi: 10.1136/gut.2004.048124

See end of article for authors' affiliations

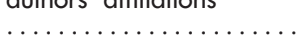

Correspondence to: Professor A Grieco, Department of Internal Medicine, Università Cattolica del Sacro Cuore, Largo Gemelli 8-00168 Roma, Italy: agrieco@ rm.unicatt.it

Revised version received 10 June 2004

Accepted for publication

19 June 2004

\begin{abstract}
Background: Several prognostic models have been developed to stage hepatocellular carcinoma (HCC) but there is no general consensus on which is the most reliable. We compared three prognostic indices (Okuda, CLIP, and BCLC scoring systems) in a large series of cirrhotic patients with HCC undergoing nonsurgical treatment in terms of their ability to classify patients into different risk groups

Methods: We retrospectively studied 268 Italian patients with HCC. A total of 146 patients were treated with ablation, 132 with percutaneous ethanol injection, and 14 with radiofrequency ablation; 103 underwent transcatheter arterial chemoembolisation and 19 had supportive care alone. Factors determining survival were analysed by univariate and multivariate analysis using the Kaplan-Meier method and Cox proportional hazard regression models. Okuda, CLIP, and BCLC scores evaluated before treatment were applied.

Results: Median survival was 25.7 months. In a multivariate analysis, portal vein thrombosis, $\alpha$ fetoprotein, total bilirubin, and tumour size were significant predictors of survival. Okuda, CLIP, and $B C L C$ scores were all able to predict survival $(p<0.001)$. They identified two, four, and six risk groups, respectively, with a median survival ranging from 27 to 19 months for Okuda, 30 to 5 months for CLIP, and 43 to 7 months for BCLC.

Conclusions: Both CLIP and BCLC scores were more effective than the Okuda score in stratifying patients into different risk groups with early-intermediate HCC. However, the BCLC scoring system gave a better prediction of prognosis in patients with disease diagnosis at a very early stage.
\end{abstract}

$\mathrm{H}$ epatocellular carcinoma (HCC) is the third most frequent cause of death from cancer in the world. Its incidence varies between 500000 and 1000000 cases a year. ${ }^{1}$ In the Western world, HCC is closely associated with hepatic cirrhosis, which is present in $70-80 \%$ of all cases. ${ }^{2-4}$ For this reason, cirrhotic patients are encouraged to undergo regular ultrasound screening. In fact, early detection, when the tumour is still small, increases the options for therapy and the likelihood of cure. ${ }^{5}{ }^{6}$ Tumour staging, which is the basis for therapeutic management, has traditionally been based on the Okuda system (table 1), which was developed 18 years ago. ${ }^{7}$ At that time, early diagnosis of HCC was relatively rare, and the staging system was therefore based on data from patients with advanced disease.

The Cancer of the Liver Italian Program (CLIP) score $^{8}$ (table 2 ) is a newer staging system that has been validated in case series from various parts of the world. ${ }^{9}$ Although its predictive power has been found to be superior to that of the Okuda system in most cases, doubts have been raised regarding its value in certain populations. ${ }^{11}$ A second alternative is the Barcelona Clinic Liver Cancer (BCLC) staging system ${ }^{12}$ (table 3 ), which, in theory, has certain advantages. Among the prognostic variables considered in BCLC staging are performance status $(P S)^{13}$ and portal hypertension, which are not taken into account in either the CLIP or Okuda system. These additional criteria should make it especially suitable for staging HCCs diagnosed early in patients with well compensated cirrhosis.

The purpose of the present study was to evaluate the influence on survival of a series of variables (clinical features, laboratory parameters, imaging data, treatment modalities) established at the time of HCC diagnosis. Cases were then retrospectively staged using the Okuda, CLIP, and BCLC systems to identify which system provided the most accurate prediction of survival.

\section{METHODS}

In this retrospective study, we analysed clinical, aetiological, and therapeutic variables in 268 asymptomatic patients with HCC observed between March 1992 and May 2002 in our institution. In most cases $(232 / 268 ; 86.6 \%)$ the tumours had been detected by abdominal ultrasound studies and/or $\alpha$ fetoprotein (AFP) assays, performed at six month intervals as part of a screening programme for cirrhotic patients aimed at early detection of HCC. Each focal lesion detected was further evaluated by multiphase spiral computed tomography (CT) or contrast enhanced magnetic resonance imaging (MRI). The presence of enhancement in the arterial phase was considered diagnostic for HCC if the lesion was larger than $2 \mathrm{~cm}$. Until the year 2000, before treatment and independently of the results of the imaging studies, whenever possible we routinely performed an ultrasound guided fine needle-biopsy study of the focal lesion. ${ }^{14}$ After 2000, ultrasound guided biopsies were confined to doubtful cases (lesions smaller

\footnotetext{
Abbreviations: HCC, hepatocellular carcinoma; CLIP, Cancer of the Liver Italian Program; BCLC, Barcelona Clinic Liver Cancer; PS, performance status; $C T$, computed tomography; MRI, magnetic resonance imaging; $H C V$, hepatitis $C$ virus; $H B V$, hepatitis $B$ virus; $P E I$ percutaneous ethanol injection; RFA, radiofrequency ablation; TACE, transcatheter arterial chemoembolisation; PVT, portal vein thrombosis; AFP, $\alpha$ fetoprotein; ROC, receiver operating characteristic
} 
Table 1 Definition of the Okuda staging system for hepatocellular carcinoma

\begin{tabular}{lll}
\hline & \multicolumn{2}{l}{ Points } \\
\cline { 2 - 3 } & 0 & 1 \\
\hline Tumour size & $<50 \%$ of liver & $>50 \%$ of liver \\
Ascites & No & Yes \\
Albumin $(\mathrm{g} / \mathrm{dl})$ & $\geqslant 3$ & $<3$ \\
Bilirubin (mg/dl) & $<3$ & $\geqslant 3$ \\
\hline $\begin{array}{l}\text { Okuda stage I, 0 points; Okuda stage II, 1 or 2 points; Okuda stage III, 3 } \\
\text { or 4 points. }\end{array}$
\end{tabular}

than $2 \mathrm{~cm}$ or without arterial enhancement on CT or MRI). ${ }^{6}$ In the remaining 36 cases (in which cases of chronic hepatitis were included), the imaging studies and/or laboratory work up had been done for other reasons.

The clinical features of the study population (mean age 65.4 years; range $42-83$ ) at the time of HCC diagnosis are summarised in table 4 . Hepatitis $\mathrm{C}$ virus (HCV) infection was the most common cause of chronic liver disease and was detected by means of third generation ELISAs for HCV antibodies and/or polymerase chain reaction assays for HCV RNA. Hepatitis B virus (HBV) infection was documented by hepatitis B surface antigen positivity and/or positivity for HBV DNA in polymerase chain reaction assays. Alcohol related liver disease was diagnosed based on a clinical history of alcohol abuse (exceeding $24 \mathrm{~g}$ /day in males or $16 \mathrm{~g}$ /day in females). ${ }^{15}$

The vast majority of patients studied had cirrhosis but most had well compensated disease according to the Child-Pugh

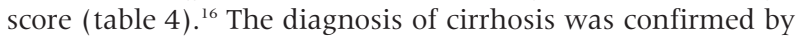
liver biopsy in 190/259 (73,4\%) cases; in the remaining 69 , cirrhosis was diagnosed based on clinical and blood chemistry findings indicative of chronic liver disease together with evidence of portal hypertension (defined by the presence of hepatic vein pressure gradient $\geqslant 10 \mathrm{~mm} \mathrm{Hg}$, splenomegaly with a platelet count $<100000 / \mathrm{mm}^{3}$, oesophageal varices, or ascites $\left.{ }^{12}\right)$. Ascites was mild in all positive cases. Portal vein thrombosis (PVT) was present in a minority of cases $(4.1 \%)$ at the time of HCC diagnosis.

Most patients (155/268) had a single lesion. Of the 113 patients with multiple nodules, 53 had two lesions and the remaining 60 had three or more lesions. The mean diameter of the largest lesion was $3.7 \mathrm{~cm}$ (range 1.5-10.7). In 249/268 patients $(93 \%)$ the final diagnosis of HCC was based on cytological and/or histological analysis of ultrasound guided fine needle biopsy specimens. ${ }^{14}$ In the remaining 19, biopsies had not been performed because imaging findings and serum AFP levels ( $>400 \mathrm{ng} / \mathrm{ml}$ ) were already considered diagnostic of HCC. ${ }^{6}$

Pretreatment staging was based on contrast enhanced CT in 241 cases; MRI was used in the remaining 27 patients who were allergic to iodised intravenous contrast agents and/or had signs of renal failure. Distant metastases were excluded in all cases based on chest $x$ ray findings and bone scans.

All of the patients referred to our institution with HCC complicating liver cirrhosis were primarily screened for liver transplantation ${ }^{17}$ or resective surgery. ${ }^{18}{ }^{19}$ The 268 patients we treated had been excluded from surgical treatment for the following reasons: Child-Pugh class $B(n=50)$, multiple lesions $(n=82)$, single lesion larger than $5 \mathrm{~cm}(n=14)$, or serious portal hypertension (defined as platelet count lower than $100000 / \mathrm{ml}$ and the presence of ascites and/or oesophageal varices) or the presence of portal vein thrombosis $(n=68)$. The remaining patients were excluded because of unacceptable surgical risk (related to the presence of chronic extrahepatic diseases or unfavourable location of the tumour in the liver parenchyma) evaluated by the consulting surgeon $(n=31)$, age older than 75 years $(n=12)$, and refusal of the patient to undergo liver resection or transplantation $(n=11)$. HCC treatments performed are shown in table 4.

Over half of the patients were treated with percutaneous ethanol injection (PEI) alone. The second most common therapeutic approach was transhepatic arterial chemoembolisation (TACE). Of the 103 patients who received this type of treatment, 39 also received PEI before or after TACE. Fourteen others were treated with radiofrequency thermal (RFA) ablation, and the remaining 19 received supportive care alone. PEI or RFA were generally reserved for patients with single lesions less than $5 \mathrm{~cm}$, or with up to three lesions, each less than $3 \mathrm{~cm}$. Transarterial chemoembolisation was applied to patients with a single lesion less than $5 \mathrm{~cm}$ which was difficult to inject with alcohol under ultrasound guidance or to patients with single lesions larger than $5 \mathrm{~cm}$ or with multiple lesions involving less than $50 \%$ of the liver parenchyma. In a small number of cases combined treatments (TACE plus PEI or RFA) were applied to patients who had residual viability of the treated lesion after one or more TACE procedures. Supportive care alone was provided to patients with multifocal bilobar disease and/or vascular invasion. PEI, RFA, and TACE were performed using standardised protocols. ${ }^{20-22}$ After treatment, patients were regularly followed up at three month intervals. In the case of local recurrence of the primary lesion or the occurrence of new nodules, a new treatment was usually planned and performed according to the same criteria used to treat the primary lesion.

\section{Statistical analysis}

Survival of patients was the single end point used to assess the performance of the different scoring systems. Length of survival was calculated from the date of HCC diagnosis to the date of death or, in the case of survivors, the date of the last follow up visit. Survival curves were estimated by the KaplanMeier method and compared, for univariate analysis, by the log rank test. For evaluation of continuous variables, patients were divided in two groups based on the median value in the study group as a whole. Significant parameters at univariate

Table 2 Definition of the cancer of the Italian program (CLIP) scoring system for hepatocellular carcinoma

\begin{tabular}{llll}
\hline & Score & & \\
\cline { 2 - 4 } & $\mathbf{0}$ & $\mathbf{1}$ & $\mathbf{2}$ \\
\hline Child-Pugh stage & $\mathrm{A}$ & $\mathrm{B}$ \\
Tumour morphology & Uninodular and extension & Multinodular and extension & Massive or extension \\
& $\leqslant 50 \%$ & $\leqslant 50 \%$ & $>50 \%$ \\
AFP (ng/ml) & $\leqslant 400$ & $\geqslant 400$ & \\
Portal vein thrombosis & No & Yes & \\
\hline AFP, $\alpha$ fetoprotein. & & & \\
Early stage (0 points); intermediate stage (1-3 points); advanced stage (4-6 points). &
\end{tabular}


Table 3 Definition of the Barcelona Clinic Liver Cancer (BCLC) staging for hepatocellular carcinoma

\begin{tabular}{|c|c|c|c|c|}
\hline \multirow[b]{2}{*}{ BCLC stage } & \multirow[b]{2}{*}{ PST } & \multicolumn{2}{|l|}{ Tumour status } & \multirow[b]{2}{*}{ Liver function status } \\
\hline & & Tumour stage & Okuda stage & \\
\hline Stage A: early $\mathrm{HCC}$ & 0 & & & \\
\hline A1 & 0 & Single, $<5 \mathrm{~cm}$ & 1 & No portal hypertension and normal bilirubin \\
\hline A2 & 0 & Single, $<5 \mathrm{~cm}$ & i & Portal hypertension and normal bilirubin \\
\hline $\mathrm{A} 3$ & 0 & Single, $<5 \mathrm{~cm}$ & i & Portal hypertension and abnormal bilirubin \\
\hline A4 & 0 & 3 tumours $<3 \mathrm{~cm}$ & $|-| \mid$ & Child-Pugh A-B \\
\hline Stage B: intermediate HCC & 0 & Large multinodular & $|-I|$ & Child-Pugh A-B \\
\hline Stage C: advanced HCC & $1-2^{*}$ & $\begin{array}{l}\text { Vascular invasion or } \\
\text { extrahepatic spread* }\end{array}$ & I-II & Child-Pugh A-B \\
\hline Stage D: end stage HCC & $3-4 \dagger$ & Any & $\mathrm{III \dagger}$ & Child-Pugh C $†$ \\
\hline
\end{tabular}

Stages A and B: all criteria should be fulfilled.

Stage C: at least one criterion; *PST 1-2 or vascular invasion/extrahepatic spread.

Stage D: at least one criterion; †PST 3-4 or Okuda stage III/Child-Pugh C.

analysis were entered into a multivariate Cox regression model to identify independent predictors of survival.

\section{Comparison of the Okuda, CLIP, and BCLC scoring systems}

The prognostic performance of each scoring system was statistically assessed, evaluating homogeneity within classification groups, discriminatory ability, and monotonicity of the gradients in the association between stages and survival rates. ${ }^{10}$ Thus we used a multistep approach.

Firstly we evaluated, at univariate analysis, the capacity of each score to distinguish categories of patient with significantly different survival (homogeneity of the score). For each score, this performance was evaluated by comparing by log rank test the survival curves of the single categories, calculated using the Kaplan-Meier method.

Next, we needed to compare the overall predictive power of survival for each scoring system to assess which gave the most accurate prediction of survival (monotonicity of the

Table 4 Clinical features at the time of diagnosis of hepatocellular carcinoma in 268 patients included in the study

\begin{tabular}{lcl}
\hline Variable & $\mathbf{n}$ & $\%$ rate \\
\hline Sex & & \\
$\quad$ Male/female & $204 / 64$ & $76.1-33.9 \%$ \\
Liver disease & & \\
$\quad$ Chronic hepatitis & 9 & $4.1 \%$ \\
$\quad$ Cirrhosis & 259 & $95.9 \%$ \\
$\quad$ Child-Pugh Class A & 209 & $80.5 \%$ \\
$\quad$ Child-Pugh Class B & 50 & $19.4 \%$ \\
Aetiology & 187 & $69.7 \%$ \\
HCV & 47 & $17.5 \%$ \\
HBV & 16 & $6.0 \%$ \\
HBV+HCV & 14 & $5.2 \%$ \\
Alcohol & 6 & $2.2 \%$ \\
Unknown & $236 / 32$ & $88.1-11.9 \%$ \\
Ascites & & \\
Absent/present & $257 / 11$ & $95.9-4.1 \%$ \\
Portal vein thrombosis & & \\
Absent/present & $155 / 113$ & $57.8-42.2 \%$ \\
No of HCC nodules & & \\
Single/multiple & $125 / 143$ & $45.9-54.1 \%$ \\
Diameter of (largest) HCC nodule & & \\
$\quad<3$ cm/ $>3$ cm & 132 & $49.2 \%$ \\
Treatment & 103 & $38.4 \%$ \\
PEI & 14 & $5.2 \%$ \\
TACE & 19 & $7.2 \%$ \\
RFA & & \\
Supportive & &
\end{tabular}

$\mathrm{HCV}$, hepatitis $\mathrm{C}$ virus; HBV, hepatitis B virus; PEl, percutaneous ethanol injection; TACE, transarterial chemoembolisation; RFA, radiofrequency ablation. score). This point was evaluated by the linear trend $\chi^{2}$ test, entering each scoring system into a Cox regression model.

Finally, to evaluate the discriminatory ability for the prediction of survival, we evaluated the accuracy of prediction of death at one, three, and five years for each scoring system. This point was evaluated calculating the area under the receiver operating characteristic (ROC) curve for each score (which is equivalent to the concordance statistic (c statistic)). ${ }^{23}$ To perform this test, patients censored before one, three, and five years were excluded from the analysis.

\section{RESULTS}

Mean duration of follow up was 32 months (range 3-130). At the end of follow up, 144/268 (53.7\%) patients had died. Survival rates at one, three, and five years were $92 \%, 46 \%$, and $24 \%$, respectively (fig 1).

\section{Univariate analysis}

Using the Kaplan-Meier method, we evaluated possible correlations between survival and 17 dichotomised variables with known values for all 268 patients (table 5). The nine factors that had a significant negative correlation with survival in the log rank test were: positivity for hepatitis B surface antigen, AFP $>22.5 \mathrm{ng} / \mathrm{ml}$, serum albumin $<3.5 \mathrm{~g} / \mathrm{dl}$, total bilirubin $>1.5 \mathrm{mg} / \mathrm{dl}$, platelet count $<100 \times 10^{9} / \mathrm{l}$, presence of ascites, presence of PVT, tumour diameter $>3.7 \mathrm{~cm}$, and presence of two or more neoplastic nodules.

\section{Multivariate analysis}

Multivariate analysis of the nine factors listed above (Cox proportional hazard regression) revealed that the following variables were independent predictors of survival: PVT, AFP levels, total bilirubin, tumour size (table 6).

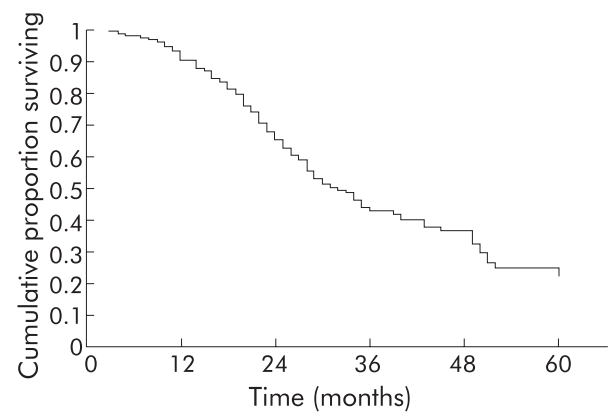

Figure 1 Overall survival of 268 patients with hepatocellular carcinoma included in the study (Kaplan-Meier method). Median survival was 25.7 months. 
Table 5 Univariate analysis of variables potentially predictive of survival in 268 patients with hepatocellular carcinoma HCC

\begin{tabular}{llllr}
\hline & Variable & $\mathbf{n}$ & $\begin{array}{l}\text { Mean survival } \\
\text { (months) }\end{array}$ & p Value \\
\hline Age (y) & $<65 />65$ & $120 / 148$ & $25.0 / 26.7$ & 0.554 \\
Sex & M/F & $208 / 60$ & $24.4 / 29.1$ & 0.111 \\
Cirrhosis & Absent/present & $9 / 259$ & $26.0 / 22.4$ & 0.291 \\
HCVAb & Negative/positive & $143 / 125$ & $26.3 / 23.9$ & 0.344 \\
HBsAg & Negative/positive & $217 / 51$ & $27.7 / 21.8$ & 0.048 \\
Alcohol abuse & Yes/no & $16 / 252$ & $24.1 / 26.3$ & 0.289 \\
AST (UI/l) & $<84 />84$ & $139 / 129$ & $27.3 / 24.3$ & 0.060 \\
ALT (UI/l) & $<85 />85$ & $133 / 135$ & $26.0 / 25.9$ & 0.912 \\
Albumin (g/dl) & $<3.5 />3.5$ & $123 / 145$ & $22.3 / 29.6$ & $<0.001$ \\
Total bilirubin (mg/dl) & $<1.5 />1.5$ & $127 / 141$ & $23.6 / 27.9$ & $<0.001$ \\
Prothrombin activity ratio (\%) & $<64 />64$ & $130 / 138$ & $24.7 / 28.1$ & 0.077 \\
Platelet count ( $\times 10^{9} /$ I) & $<100 />100$ & $133 / 135$ & $22.0 / 26.5$ & $\mathbf{0 . 0 4 1}$ \\
AFP (ng/ml) & $<22.5 />22.5$ & $145 / 123$ & $29.4 / 22.0$ & $<\mathbf{0 . 0 0 1}$ \\
Ascites & No/yes & $234 / 34$ & $26.6 / 19.3$ & $<\mathbf{0 . 0 0 1}$ \\
PVT & No/yes & $257 / 11$ & $26.3 / 9.0$ & $<\mathbf{0 . 0 0 1}$ \\
Tumour size (cm) & $<3.7 />3.7$ & $125 / 143$ & $26.3 / 21.9$ & $<\mathbf{0 . 0 0 1}$ \\
No of lesions & Single/multiple & $155 / 113$ & $27.8 / 22.7$ & $<\mathbf{0 . 0 0 1}$ \\
\hline
\end{tabular}

HCVAb, antibodies to hepatitis $C$ virus; $H B s A g$, hepatitis B surface antigen; AST, aspartate aminotransferase; ALT, alanine aminotransferase; AFP, $\alpha$ fetoprotein; PVT, portal vein thrombosis.

\section{Staging systems as predictors of survival in the study population}

Table 7 shows the results of retrospective staging of the 268 patients using the Okuda, CLIP, and BCLC systems, with median survival times and death rates. There was a significant correlation between survival and tumour stage assigned using all three systems (Okuda $\mathrm{p}<0.001$; CLIP $\mathrm{p}<0.001 ;$ BCLC $\mathrm{p}<0.001$ ). With all three methods the vast majority of cases were classified as early stage tumours-that is, Okuda stage I (190/268; 70\%); CLIP stages 0-1 (211/268; $78 \%)$; and BCLC stage A $(224 / 268 ; 82 \%)$-and none of the patients had advanced disease (Okuda stage III, CLIP stages 5-6, or BCLC stage D). Median survival of the 190 patients with Okuda stage I tumours (27.2 months) was significantly longer than that of the 78 stage II patients (19.2 months) $(\mathrm{p}<0.001)$.

BCLC stages in our population ranged from Al to $\mathrm{C}$ (table 7). Significant differences were found among survival rates of subgroups with stage $\mathrm{A}, \mathrm{B}$, and $\mathrm{C}$ tumours (A $v$ B $\mathrm{p}<0.01 ; \mathrm{B} v \mathrm{C} \mathrm{p}=0.04)$. The Al subgroup, which included patients with single HCC nodules $<3 \mathrm{~cm}$ in diameter and no signs of portal hypertension, was characterised by a significantly longer survival compared with that of the stage A2 subgroup $(p=0.04)$. Survival in the BCLC A2 subgroup was also better than that of stage $A 3(p=0.05)$, but there were no significant differences between the A3 and A4

Table 6 Cox proportional hazards model: analysis of pretreatment parameters that were significant predictors of survival in the univariate analysis

\begin{tabular}{lll}
\hline $\begin{array}{l}\text { Variables included } \\
\text { (reference) }\end{array}$ & Odds ratio $(95 \% \mathrm{CI})$ & p Value \\
\hline PVT (present) & $4.07(1.48-11.17)$ & 0.006 \\
AFP (high) & $2.25(1.33-3.78)$ & 0.002 \\
Bilirubin (high) & $1.95(1.15-3.31)$ & 0.012 \\
Tumour size (high) & $1.86(1.12-3.09)$ & 0.016 \\
Albumin (low) & $1.65(0.95-2.86)$ & 0.075 \\
Ascites (present) & $1.91(0.92-3.79)$ & 0.082 \\
Multiple lesions & $1.47(0.85-2.54)$ & 0.160 \\
Platelets (low) & $1.38(0.84-2.29)$ & 0.199 \\
HBsAg+ & $1.33(0.76-2.34)$ & 0.304 \\
\hline
\end{tabular}

PVT, portal vein thrombosis; AFP, $\alpha$ fetoprotein; $\mathrm{HBs} A g$, hepatitis $B$ surface antigen. subgroups $(p=0.25)$. However, the differences in survival between BCLC Al-A4 may not be reliable because of the small sample size.

The CLIP stages assigned in the study population ranged from 0 to 4 (table 7 ), and log rank analysis revealed significant differences in the duration of survival for each subgroup with the exception of stages 2 and 3 (CLIP $0 v 1$ $\mathrm{p}<0.01 ; 1 \vee 2 \mathrm{p}=0.01 ; 2 \vee 3 \mathrm{p}=0,11 ; 3 \vee 4 \mathrm{p}=0.04)$. Patients in CLIP stage 4 had a particularly unfavourable prognosis, with a median survival of 4.8 months from diagnosis.

Both the CLIP and BCLC systems identified distinct subgroups with a different prognosis within each Okuda stage. In particular, BCLC restaging of the 190 patients with Okuda stage I HCCs revealed a subgroup of 30 patients whose tumours were classified as BCLC stage Al and who showed a mean survival longer than that observed in the whole group (43.4 months $v 27.2$ months). In contrast, the seven patients classified as BCLC stage B and the three patients classified as BCLC stage $C$ had a worse prognosis than that predicted by the median of the group as a whole: 17.6 and 7.4 months, respectively, versus 27.2 months (fig 2A). Similar results were seen when the 78 Okuda stage II patients (mean survival 19.4 months) were reclassified with the BCLC system: eight of these cases were stage $C$ tumours and mean survival of this group was only seven months (fig $2 \mathrm{~B}$ ). When considering CLIP restaging of Okuda I patients, no significant survival differences were found among subgroups (fig 3A). Within the group of Okuda II patients, only the CLIP 0 subgroup had a significantly longer survival than the other groups (fig 3B).

When entered into a Cox regression model, the BCLC score showed slightly better performance in prediction of overall survival compared with the CLIP and Okuda scores (table 8). BCLC showed a better performance compared with CLIP and Okuda even when patients treated by PEI and TACE (table 8) were considered separately.

Discriminatory ability for death at one, three, and five years, evaluated by ROC curve area analysis, was slightly higher for BCLC compared with CLIP and Okuda. While no significant difference was found at one year, BCLC and CLIP showed a significantly better discriminatory ability at three and five years compared with the Okuda score. No significant difference was found comparing BCLC and CLIP scores at one, three, and five years (table 9). 


\begin{tabular}{|c|c|c|c|c|c|}
\hline Staging system & $\mathrm{n}$ & $\begin{array}{l}\text { Median } \\
\text { survival }\end{array}$ & $\begin{array}{l}\text { Deaths } \\
\text { (n) }\end{array}$ & $\begin{array}{l}\text { Deaths } \\
\text { (\% rate) }\end{array}$ & p Value \\
\hline Okuda & & & & & $<0.001$ \\
\hline 1 & 190 & 27.5 & 70 & 36.8 & \\
\hline ॥ & 78 & 19.4 & 54 & 69.2 & \\
\hline III & 0 & - & - & - & - \\
\hline CLIP & & & & & $<0.001$ \\
\hline 0 & 129 & 30.1 & 45 & 34.7 & \\
\hline 1 & 82 & 25.0 & 41 & 50.2 & \\
\hline 2 & 33 & 17.7 & 19 & 57.6 & \\
\hline 3 & 19 & 16.8 & 15 & 78.9 & \\
\hline 4 & 5 & 4.8 & 4 & 80.0 & \\
\hline 5 & 0 & - & - & - & \\
\hline 6 & 0 & - & - & - & \\
\hline BCLC & & & & & $<0.001$ \\
\hline $\mathrm{Al}$ & 30 & 43.4 & 6 & 20.4 & \\
\hline A2 & 68 & 28.9 & 22 & 32.1 & \\
\hline A3 & 33 & 25.4 & 16 & 48.5 & \\
\hline A4 & 93 & 22.3 & 46 & 49.4 & \\
\hline B & 33 & 17.6 & 27 & 81.8 & \\
\hline C & 11 & 7.4 & 7 & 63.0 & \\
\hline D & 0 & - & - & - & \\
\hline
\end{tabular}
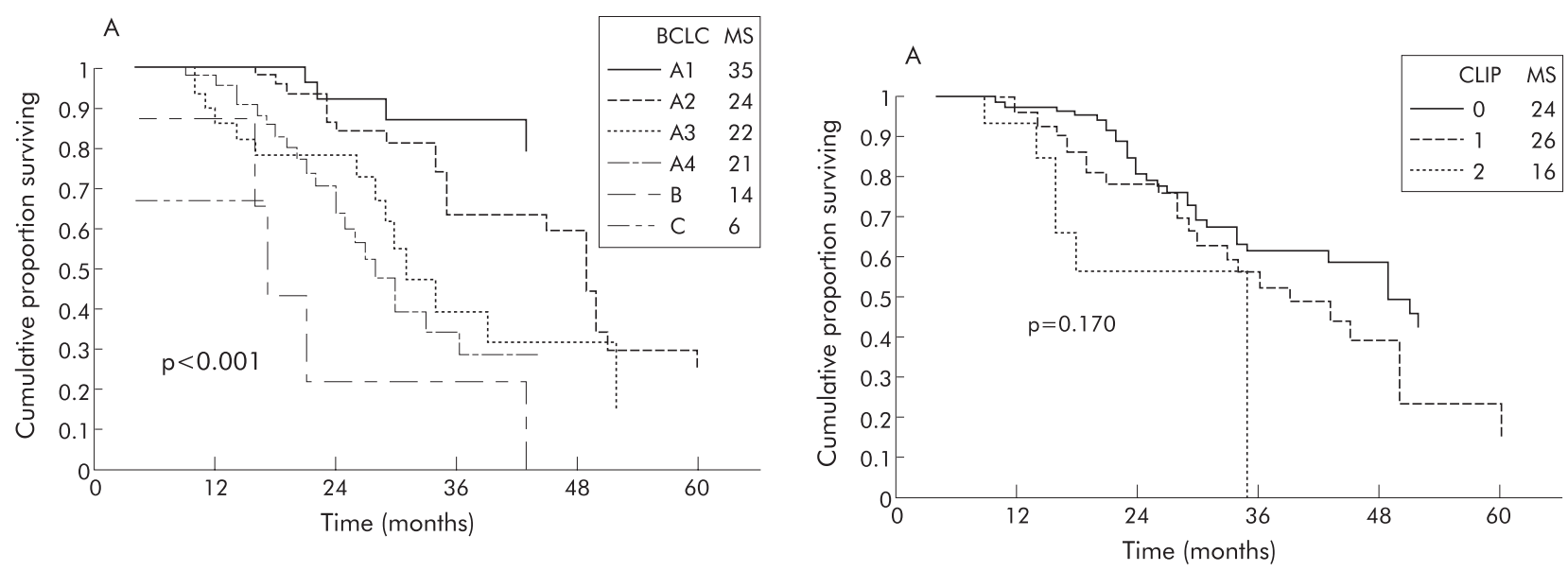

B
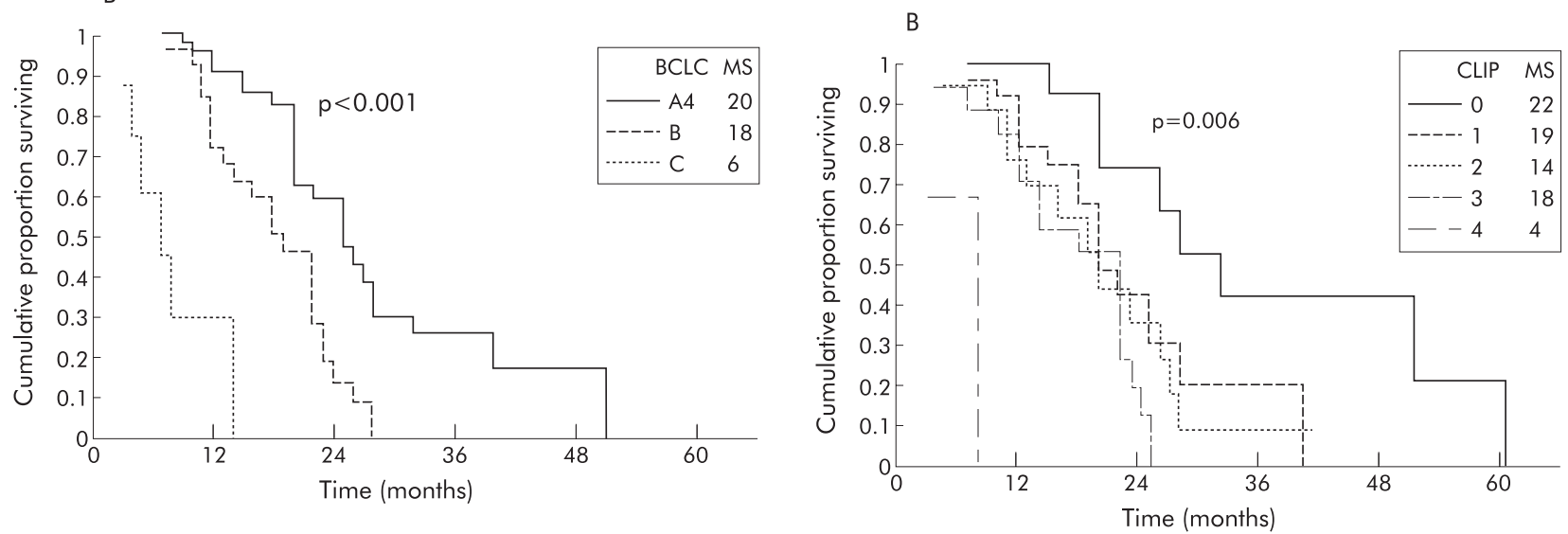

Figure 2 (A, B) Kaplan-Meier estimated survival curves by Barcelona Clinic Liver Cancer (BCLC) score of patients classified as stage I and stage II using the Okuda score system. (A) Okuda I: BCLC A1 $v$ A2 $p=0.003 ; A 2 v A 3 p=0.035 ; A 3 v A 4 p=0.303 ; A 4 \vee B p=0.139 ; B v$ $C p=0.932$. (B) Okuda II: BCLC A4 $\vee B p<0.001 ; B \vee C p=0.044$. MS, median survival (months).

Figure 3 (A, B) Kaplan-Meier estimated survival curves by Cancer of the Liver Italian Program (CLIP) score of patients classified as stage I and stage II using the Okuda score system. (A) Okuda I: CLIP 0 v $1 p=0.102$; $1 v 2 p=0.080$. (B) Okuda II: CLIP 0 v $1 p=0.013 ; 1 v 2 p=0.682 ; 2 v$ $3 p=0.271 ; 3 \vee 4 p=0.208$. MS, median survival (months). 
Table 8 Performance evaluation of the Okuda, CLIP, and BCLC staging systems

\begin{tabular}{|c|c|c|c|}
\hline Staging system & $\chi^{2}$ value & -Log likelihood & p Value \\
\hline \multicolumn{4}{|l|}{ All patients } \\
\hline Okuda & 50.4 & 1102.6 & $<0.001$ \\
\hline CLIP & 76.8 & 1082.6 & $<0.001$ \\
\hline BCLC & 89.9 & 1049.3 & $<0.001$ \\
\hline \multicolumn{4}{|l|}{ PEI treatment } \\
\hline Okuda & 3.9 & 279.9 & 0.047 \\
\hline CLIP & 9.3 & 275.7 & 0.002 \\
\hline $\mathrm{BCLC}$ & 12.8 & 270.7 & 0.001 \\
\hline \multicolumn{4}{|l|}{ TACE treatment } \\
\hline Okuda & 22.1 & 485.5 & $<0.001$ \\
\hline CLIP & 10.4 & 496.1 & 0.001 \\
\hline $\mathrm{BCLC}$ & 26.2 & 477.7 & $<0.001$ \\
\hline \multicolumn{4}{|c|}{$\begin{array}{l}\text { Data are for the whole population and for PEI treatment and TACE } \\
\text { treatment subgroups. } \\
\text { In the same group of patients, higher } \chi^{2} \text { values and lower -log likelihood } \\
\text { values were associated with better performance of the score. } \\
\text { CLIP, Cancer of the Liver Italian Program; BCLC, Barcelona Clinic Liver } \\
\text { Cancer; PEI, percutaneous ethanol injection; TACE, transcatheter arterial } \\
\text { chemoembolisation. }\end{array}$} \\
\hline
\end{tabular}

\section{DISCUSSION}

The prognosis for cirrhotic patients who develop HCC continues to be poor, with a five year survival rate of $6 \%$ in the USA. ${ }^{1}$ The various forms of treatment available for these tumours are effective in improving survival only when the disease is diagnosed at an early stage (that is, when tumour diameter is less than $5 \mathrm{~cm}$ and liver function is still normal). For patients with larger tumours and decompensated cirrhosis (Child-Pugh class B or C), no treatment appears to be capable of prolonging survival. ${ }^{24}$ Ultrasound screening of patients with chronic hepatitis and cirrhosis has increased the frequency of preclinical diagnosis of $\mathrm{HCC}^{25}$ and consequently curative therapy (that is, resection, liver transplantation, PEI, RFA, and TACE) is considered possible in a growing number of cases.

Tumour staging at the time of diagnosis is essential to identify these cases and decide which type of therapy is the most appropriate. The Okuda system is proving to be inadequate for today's HCC cases, particularly those that are diagnosed early. Designed for use in a population with relatively advanced neoplastic disease, the Okuda system's current shortcomings include a lack of distinction between uni- and multifocal tumours and its neglect of factors such as AFP levels, PVT, local-regional lymph node involvement and PS, whose prognostic significance has been unequivocally confirmed in both surgical and non-surgical series. ${ }^{26-30}$

In recent years, several alternative staging systems have been proposed ${ }^{8111^{1-33}}$ but there is still a lack of consensus regarding their relative accuracies and applicability. Significant biological variability related to the various aetiological/pathogenetic aspects of these tumours and our incomplete understanding of their natural history are two of the factors complicating the staging process. ${ }^{34}$

The system proposed by the Cancer of the Liver Italian Program (CLIP) in 1998 was based on a retrospective study ${ }^{8}$ and was subsequently prospectively validated..$^{35}$ The CLIP studies identified five independent prognostic variables: Child-Pugh class of cirrhosis, tumour size, number of lesions, presence/absence of PVT, and serum levels of AFP. On the whole, CLIP stages have proved to be more accurate than the Okuda classification scheme in the prediction of survival, particularly in early stage disease. ${ }^{8}$ The validity of this system has been confirmed in two large retrospective studies in Canada and Japan ${ }^{9}{ }^{10}$ but in the largest series evaluated thus far (906 Patients in China), CLIP staging displayed very low correlation with survival. ${ }^{11}$ This discrepancy may be related to
Table 9 Discriminatory ability for death at one, three, and five years, evaluated by receiver operating characteristic (ROC) curve area, for Okuda, CLIP, and $\mathrm{BCLC}$ scores

\begin{tabular}{|c|c|c|}
\hline Staging system & ROC curve area & $95 \% \mathrm{Cl}$ \\
\hline \multicolumn{3}{|c|}{1 year death $(24 / 241)$} \\
\hline Okuda & 0.702 & $0.640-0.759$ \\
\hline CLIP & 0.782 & $0.724-0.832$ \\
\hline $\mathrm{BCLC}$ & 0.816 & $0.762-0.863$ \\
\hline \multicolumn{3}{|c|}{3 year death $(104 / 153)$} \\
\hline Okuda & 0.684 & $0.611-0.750$ \\
\hline CLIP & 0.730 & $0.660-0.793$ \\
\hline $\mathrm{BCLC}$ & 0.779 & $0.712-0.836$ \\
\hline \multicolumn{3}{|c|}{5 year death $(118 / 134)$} \\
\hline Okuda & 0.669 & $0.588-0.743$ \\
\hline CLIP & 0.726 & $0.648-0.795$ \\
\hline $\mathrm{BCLC}$ & 0.731 & $0.653-0.799$ \\
\hline \multicolumn{3}{|c|}{$\begin{array}{l}\text { Patients censored before one, three, and five years were respectively } \\
\text { excluded from the analysis. } \\
\text { Higher ROC curve area values indicate a better discriminatory ability of } \\
\text { the score. } \\
\text { CLIP, Cancer of the Liver Italian Program; BCLC, Barcelona Clinic Liver } \\
\text { Cancer. }\end{array}$} \\
\hline
\end{tabular}

the fact that the majority of Chinese patients had HBV related liver disease whereas HCV was the most common risk factor in the Canadian and Japanese studies (as well as in those conducted in other Western countries).

The BCLC staging system was developed based on a retrospective analysis of various studies of HCC patients with early, intermediate, and advanced-terminal disease, which attempted to identify prognostically relevant variables for each group. ${ }^{12}$ For patients with early stage disease, survival was negatively correlated with portal hypertension and bilirubin levels $>1.5 \mathrm{mg} / \mathrm{dl}$; for intermediate stages, the significant variable was a large multinodular tumour; and for advanced disease, deterioration of PS and the presence of vascular infiltration. These variables were used to supplement those included in the Okuda staging system. To our knowledge, only two attempts have been made to compare the CLIP and BCLC systems. In the first study, both scores displayed prognostic values inferior to that of oestrogen receptor status $^{36}$; in the latter, BCLC proved to have a higher prognostic power than CLIP in 187 HCC patients undergoing surgical or non-surgical treatment. ${ }^{37}$

In our study, none of the patients had undergone surgical resection or liver transplantation, and all had received nonsurgical forms of treatment. Furthermore, all of the tumours had been diagnosed before they had provoked symptoms as the vast majority of the patients had been subjected to periodic ultrasound screening for early detection of HCC. Mean duration of survival in the total population (25.7 months) was comparable (20 months) with that reported in a population similar to ours for the percentage of tumours diagnosed at the preclinical stages and for the number of patients with confirmed cirrhosis at the time of diagnosis. ${ }^{8}$ The high prevalence of cirrhosis in ours and the CLIP study populations ${ }^{8}$ is probably largely responsible for the lower survival rates with respect to those reported by Ueno et al (mean 37.7 months) in a group that included a much higher percentage $(19.2 \%)$ of patients with no evidence of cirrhosis at the time of HCC diagnosis. ${ }^{10}$

Multivariate analysis showed that independent predictors of survival were mostly related to the cancer itself (tumour diameter $<3 \mathrm{~cm}$, absence of PVT, and low serum AFP) while only a low bilirubin serum level was significantly related to survival among the parameters of liver function. CLIP and BCLC restaging of these patients was possible as information was available in all cases for all of the variables included in both systems, including PS. In fact, all of our patients had a 
PS of 0 , reflecting the absence of symptoms at the time of diagnosis. Both the BCLC and CLIP systems proved to be superior to the Okuda system, distinguishing within each Okuda stage various subgroups that were distinct in terms of survival. On the whole, when the two new systems were compared, BCLC proved to be the more accurate predictor of survival, even if its discriminating power at three and five years did not differ significantly from that provided by the CLIP score.

Moreover, in our patients, BCLC staging offered two important advantages: better differentiation of early stage HCCs and inclusion in the score of PVT presence. In fact, stage A included four subgroups that were significantly different in terms of survival. The best prognosis was associated with stage Al cases (mean survival 43.4 months) and this was probably due to the fact that none of the Al patients had tumours $>3 \mathrm{~cm}$, portal hypertension, or elevated bilirubin levels - each of which is known to have a negative influence on survival in both surgical and nonsurgical series. ${ }^{30} 3839$ Interestingly, both tumour size and bilirubin level were independent predictors of survival, even in our series. In contrast, almost half $(53 / 129)$ of the CLIP 0 patients had lesions $>3 \mathrm{~cm}$, and in 21 cases the tumours were $>4 \mathrm{~cm}$. Furthermore, findings indicative of portal hypertension (which is not considered in CLIP staging) were present in $163(60.8 \%)$ patients. Consequently, CLIP stage 0 was associated with a mean survival of only 30.1 months. Other groups have reported considerably better survival values for patients of this type, ranging from 42.5 to 68.7 months. ${ }^{8}{ }^{10}$ The difference is probably related to the inclusion in the latter two studies of a number of patients who underwent surgical resection.

The second advantage of BCLC staging is that it reflects the presence or absence of PVT. In our experience, survival in patients with PVT at the time of diagnosis is invariably limited, and this effect is independent of the presence of other prognostically negative factors. In patients with HCC, PVT is generally indicative of neoplastic invasion of the vascular compartment. ${ }^{40}$ In the present study, eight of the 11 patients with PVT died within eight months after diagnosis. The outcome of the other three cases is unknown as the patients were lost to follow up 3-4 months after diagnosis. BCLC staging classified all 11 of these patients as stage C (mean survival 7.4 months). In contrast, with the CLIP system, seven of the PVT patients were classified as stage 2 or 3 , both of which were associated with considerably longer survival (17.7 and 16.8 months, respectively). The remaining four were classified as stage 4 .

Several follow up studies have shown that even mild elevations in serum AFP levels predict a worse prognosis, regardless of whether the patient is treated with surgery or PEI. ${ }^{30}{ }^{41}$ AFP levels are one of the variables considered in the CLIP system but are not included in the BCLC. In terms of prognostic accuracy, this difference does not appear to be important. In fact, the CLIP definition of an AFP elevation is $>400 \mathrm{ng} / \mathrm{ml}$. While this cut off is widely accepted as diagnostic for HCC, only 19/268 (7\%) of our patients had levels this high at the time of diagnosis, and eight of these also had PVT, which automatically placed them in BCLC stage C.

In conclusion, in this series of patients with asymptomatic HCCs diagnosed at the early to intermediate phases and treated with non-surgical modalities, both the CLIP and BCLC systems provided more precise estimates of survival than the Okuda system. The BCLC system appeared to be more accurate than the CLIP score in identifying prognostically favourable cases (small tumours $<3 \mathrm{~cm}$ in patients with well compensated liver function and no portal hypertension) as well as those in whom survival was likely to be limited (due to the presence of macroscopically evident vascular invasion).

Authors' affiliations

A Grieco, M Pompili, G Caminiti, L Miele, M Covino, B Alfei,

G L Rapaccini, G Gasbarrini, Department of Internal Medicine, Policlinico Universitario A Gemelli, Università Cattolica del Sacro Cuore, Roma, Italy

Conflict of interest: None declared.

\section{REFERENCES}

1 Bosch FX. Global epidemiology of hepatocellular carcinoma. In: Okuda K, Tabor E, eds. Liver cancer. New York: Churchill Livingston, 1997:13-28.

2 Poynard T, Bedossa P, Opolon P. Natural history of liver fibrosis progression in patients with chronic hepatitis C. The OBSVIRC, METAVIR, CLINIVIR, and DOSVIRC groups. Lancet 1997;349:825-32.

3 Fattovich G, Brollo L, Giustina G, et al. Natural history and prognostic factors for chronic hepatitis type B. Gut 1991;32:294-8.

4 Trevisani F, D'Intino PE, Morselli-Labate AM, et al. M. Serum alphafetoprotein for diagnosis of hepatocellular carcinoma in patients with chronic liver disease: influence of HBsAg and anti-HCV status, J Hepatol 2001;34:570-5.

5 Tanaka S, Kitamura T, Nakanishi K, et al. Effectiveness of periodic checkup by ultrasonography for the early diagnosis of hepatocellular carcinoma. Cancer 1990;66:2210-14.

6 Bruix J, Sherman M, Llovet JM, et al. EASL Panel of Experts on HCC. Clinical management of hepatocellular carcinoma. Conclusions of the Barcelona-2000 EASL conference. European Association for the Study of the Liver. J Hepatol 2001;35:421-30.

7 Okuda K, Ohtsuki T, Obata H, et al. Natural history of hepatocellular carcinoma and prognosis in relation to treatment. Study of 850 patients. Cancer 1985;56:918-28.

8 The Cancer of Liver Italian Program (CLIP) Investigators. A new prognostic system for hepatocellular carcinoma: a retrospective study of 435 patients. Hepatology 1998;28:751-5.

9 Levy I, Sherman M. Liver cancer study group of the University of Toronto. Staging of hepatocellular carcinoma: assesment of the CLIP, Okuda, and Child-Pugh staging system in a cohort of 257 patients in Toronto, Gut 2002;50:881-5.

10 Ueno S, Tanabe G, Sako K, et al. Discrimination value of the new Western prognostic system (CLIP score) for hepatocellular carcinoma in 662 Japanese patients. Hepatology 2001;34:529-34.

11 Leung T, Tang A, Zee B, et al. Construction of the Chinese University prognostic index (CUPI) for hepatocellular carcinoma and comparison with the TNM staging system, the Okuda staging system, and the Cancer of the Liver Italian program staging system. Cancer 2002;94:1760-9.

12 Llovet JM, Brù C, Bruix J. Prognosis of hepatocellular carcinoma: the BCLCstaging classification. Semin Liver Dis 1999;19:329-38.

13 Sorensen JB, Klee M, Palshof T, et al. Performance status assessment in cancer patients. An inter-observer variability study. Br J Cancer 1993;67:773-5.

14 Rapaccini GL, Pompili M, Caturelli E, et al. Ultrasound-guided fine-needle biopsy of hepatocellular carcinoma: comparison between smear cytology and microhistology. Am J Gastroenterol 1994;89:898-902.

15 Becker U, Deis A, Sorensen TI, et al. Prediction of risk of liver disease by alcohol intake, sex, and age: a prospective population study. Hepatology 1996;23:1025-9.

16 Pugh R, Murray-Lyon IM, Dawson JL, et al. Transection of the oesophagus for bleeding oesophageal varices. Br J Surg 1973;60:646-9.

17 Mazzaferro V, Regalia E, Doci R, et al. Liver transplantation for the treatment of small hepatocellular carcinomas in patients with cirrhosis. N Engl J Med 1996;334:693-9.

18 Bruix J. Treatment of hepatocellular carcinoma. Hepatology 1997;25:259-62.

19 Ryder SD. Guidelines for the diagnosis and treatment of hepatocellular carcinoma (HCC) in adults. Gut 2003;52(suppl):iii 1-8.

20 Pompili M, Rapaccini GL, de Luca F, et al. Risk factors for intrahepatic recurrence of hepatocellular carcinoma in cirrhotic patients treated by percutaneous ethanol injection. Cancer 1997;79:1501-8.

21 Livraghi T, Goldberg SN, Lazzaroni S, et al. Hepatocellular carcinoma: radiofrequency ablation of medium and large lesions. Radiology 2000;214:761-8.

22 Grieco A, Marcoccia S, Miele L, et al. Transarterial chemoembolization (TACE) for unresectable hepatocellular carcinoma in cirrhotics: functional hepatic reserve and survival. Hepatogastroenterology 2003:50:207-12.

23 Hanley JA, MCNeil BJ. The meaning and use of the area under a receiver operating caracteristic (ROC) curve. Radiology 1982;143:29-36.

24 Johnson PJ. Hepatocellular carcinoma: is current therapy really altering outcome? Gut 2002;51:459-62

25 Solmi L, Primerano AM, Gandolfi L. Ultrasound follow-up of patients at risk for hepatocellular carcinoma: results of a prospective study on 360 cases. Am J Gastroenterol 1996;91:1189-94.

26 Poon RT, $\mathrm{Ng}$ IO, Fan ST, et al. Clinicopathologic features of long-term survivors and disease-free survivors after resection of hepatocellular carcinoma: a study of a prospective cohort. J Clin Oncol 2001;19:3037-44.

27 The Liver Cancer Study Group of Japan. Predictive factors for long term prognosis after partial hepatectomy for patients with hepatocellular carcinoma in Japan. Cancer 1994;74:2772. 
28 Terris B, Laurent-Puig P, Belghitti J, et al. Prognostic influence of clinicopathologic features, DNA-ploidy, CD44H and p53 expression in a large series of resected hepatocellular carcinoma in France. Int $J$ Cancer 1997;74:614-19.

29 Hanazaki K, Kajikawa S, Koide N, et al. Prognostic factors after hepatic resection for hepatocellular carcinoma with hepatitis $C$ viral infection: univariate and multivariate analysis. Am J Gastroenterol 2001;96:1243-50.

30 Pompili M, Rapaccini GL, Covino M, et al. Prognostic factors for survival in patients with compensated cirrhosis and small hepatocellular carcinoma after percutaneous ethanol injection therapy. Cancer 2001;92:126-35.

31 Schoniger-Hekele M, Muller C, Kutilek M, et al. Hepatocellular carcinoma in central Europe: prognostic features and survival. Gut 2001;48:103-8.

32 Chevret S, Trinchet JC, Mathieu D, et al. A new prognostic classification for predicting survival in patients with hepatocellular carcinoma. Groupe d'Etude et de Traitement du Carcinome Hepatocellulaire. J Hepatol 1999;31:133-41.

33 Liver Cancer Study Group of Japan. The general rule of the clinical and pathologica study of primary liver cancer, 3rd edn. Tokyo: Kanehara \& Co. Ltd, 1992.

34 Johnson P, Bruix J. Hepatocellular carcinoma and the art of prognostication. J Hepatol 2000;33:1006-8.
35 The Cancer of Liver Italian Program (CLIP) Investigation. Prospective validation of the CLIP score: a new prognostic system for patients with hepatocellular carcinoma. Hepatology 2000;31:840-5.

36 Villa E, Colantoni A, Camma C, et al. Estrogen receptor classification for hepatocellular carcinoma: comparison with clinical staging systems. J Clin Oncol 2003;21:441-6.

37 Cillo U, Bassanello M, Vitale A, et al. The critical issue of hepatocellular carcinoma prognostic classification: which is the best tool available? J Hepatol 2004:40:124-31

38 Bruix J, Castells A, Bosch J, et al. Surgical resection of hepatocellular carcinoma in cirrhotic patients: prognostic value of preoperative portal pressure. Gastroenterology 1996;111:1018-22.

39 Mondazzi L, Bottelli R, Brambilla G, et al. Transarterial oily chemoembolization for the treatment of hepatocellular carcinoma: a multivariate analysis of prognostic factors. Hepatology 1994;19:1115-23.

40 Cedrone A, Rapaccini GL, Pompili, et al. Portal vein thrombosis complicating hepatocellular carcinoma. Value of ultrasound-guided fine-needle biopsy of the thrombus in the therapeutic management. Liver 1996;16:94-8.

41 Lencioni R, Bartolozzi C, Caramella D et al. Treatment of small hepatocellular carcinoma with percutaneous ethanol injection. Analysis of prognostic factors in 105 Western patients. Cancer 1995;76:1737-46.

\section{EDITOR'S QUIZ: GI SNAPSHOT}

Postprandial abdominal pain, megaloblastic anaemia, and severe weight loss in an 80 year old man

\section{Clinical presentation}

An 80 year old man with no previous medical history was referred to our hospital with progressive weight loss of $24 \mathrm{~kg}$ in the past 12 months. There were no episodes of diarrhoea, fever, night sweats, nausea, or vomiting in the past 12 months. Physical examination revealed a significant reduction in the patient's general and nutritional status (weight $56 \mathrm{~kg}$, size $178 \mathrm{~cm}$, body mass index $17.6 \mathrm{~kg} / \mathrm{m}^{2}$ ), symmetrical oedema of the forearms and lower legs, and pleural effusions. Pathological laboratory findings included: erythrocyte sedimentation rate $82 \mathrm{~mm} / \mathrm{h}$; C reactive protein $3.8 \mathrm{mg} / \mathrm{dl}$ (normal $0.8 \mathrm{mg} / \mathrm{dl}$ ); serum protein $5.6 \mathrm{~g} / \mathrm{dl}$ (normal 6.5-8.5 g/dl); serum albumin $1.8 \mathrm{~g} / \mathrm{dl}$ (normal 3.6-5.0 g/dl); serum iron $3.3 \mu \mathrm{mol} / \mathrm{l}$ (normal 10.6-26.0 $\mu \mathrm{mol} / \mathrm{l}$ ); haemoglobin $10.7 \mathrm{~g} / \mathrm{dl}$ (normal 14-18 g/dl); MCV $119.7 \mathrm{fl}$ (normal 86-101 fl); and vitamin $B_{12} 74 \mathrm{pg} / \mathrm{ml}$ (normal 199-730 pg/ml). Computed tomography scans of the chest and abdomen showed symmetrical pleural effusions but no signs of a solid tumour. Upper endoscopy, including histological examination of the stomach and duodenum, revealed no pathological findings. Total colonoscopy and ileoscopy demonstrated a diverticulosis of the sigmoid colon. Histological examination of colonic biopsies did not reveal any pathological findings. Radiographic enteroclysis was performed (fig 1).

\section{Question}

What is the diagnosis? What additional investigations are necessary?

See page 436 for answer

This case is submitted by:

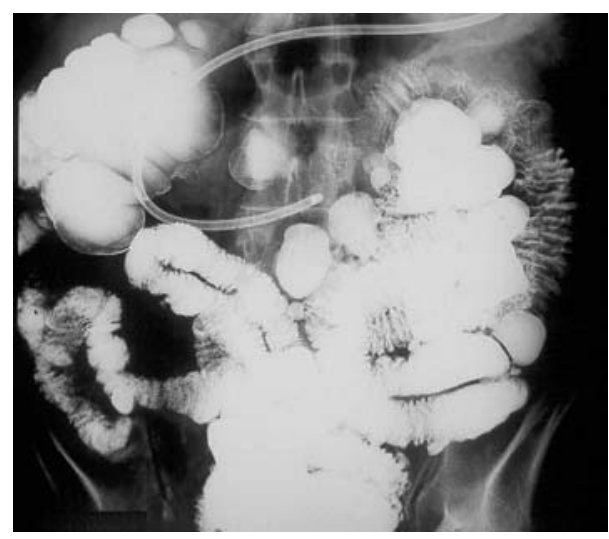

Figure 1 Radiographic enteroclysis of the patient.

A U Dignass, M E Pascu, A B Roznowski, B Wiedenmann Department of Internal Medicine, Division of Hepatology and Gastroenterology, Charité Medical School-Campus Virchow Clinic, Berlin, Germany

Correspondence to: Dr M E Pascu, Charité Universitätsmedizin BerlinCampus Virchow, Medizinische Klinik m S Hepatologie und Gastroenterologie, Augustenburger Platz 1, 13353 Berlin, Germany; axel.dignass@charite.de

doi: 10.1136/gut.2004.049262 